\title{
Pilots Psychophysiological Condition Assesment
}

\section{Michaela Šerlová ${ }^{,}$, Sarah Van den Bergh ${ }^{1 *}$, Vladimír Socha ${ }^{1,2}$, Lenka Hanáková ${ }^{1,2}$}

\author{
${ }^{1}$ Department of Air Transport, Faculty of Transportation Sciences, Czech Technical University in Prague, Prague, \\ Czech Republic \\ ${ }^{2}$ Department of Information and Communication Technologies in Medicine, Faculty of Biomedical Engineering, Czech \\ Technical University in Prague, Prague, Czech Republic \\ *Corresponding author: Czech Technical University in Prague, Faculty of Transportation Sciences, Department of Air \\ Transport, Horská 3, 12803 Prague, Czech Republic, Email: vandesar@fd.cvut.cz
}

\begin{abstract}
Due to increasing safety standards in air transport, the emphasis is put on human factors in this domain. This regards especially the improvement of piloting precision during flight training and the elimination of internal and external influences with negative effect on pilots. This paper is focused on evaluation of pilot's psychophysiological state during flight training on a simulator and in an aircraft and also on pilot's reaction on transfer from analogue to digital visual presentation of avionic data. The best indicator for evaluating human's psychophysiological condition could be a heart rate because of its descriptive activity of human heart and its psychophysiological character based on sympathovagal balance of autonomic nervous system. This paper is oriented towards description and comparison of the most common methods for physiological parameters assessment, i.e. time and frequency domain analysis and non-parametric methods analysis. The paper also describes a wavelet transform. The results show that not every parameter resulting from each analysis could be a good indicator describing pilot's stress. Results also show which parameters might work as good indicators of pilot's stress - those are LF/HF ratio and parameters measured by wavelet transform. Best way to define pilot's stress on simulators in real time indicates to be wavelet.
\end{abstract}

\section{Keywords}

Air transportation - Flight simulator - Heart rate - Variability - Pilots psychophysiological state - Wavelet transform

\section{Introduction}

Due to increasing safety standards in air transport the emphasis is focused on human factors in this domain. This regards especially the improvement of piloting precision during flight training and the elimination of internal and external influences with negative effect on pilots. But in last years the International Civil Aviation Organization (ICAO) and the Eu- ropean Aviation Safety Agency (EASA) create the Alternative Training Qualification Program (ATQP) and Evidence-based Training (EBT) [1,2]. Both of these programs focus not only on improving the functioning of the general structure of air carriers but also on identifying the physiological functions of crew members using different tools. Both of the alternative programmes are oriented to the specific needs of the airline, the diversity of the crew members and especially the pilots. 
In that case training itself and the standards of training are increasing. But still $60 \%$ to $80 \%$ of accidents were caused by human errors and $50 \%$ of them directly by pilots [3]. In spite of the advanced technology it is necessary to determine what exactly causes the accidents. It seems that the level of pilot training and the level of pilot's aviation experience affected his/her mental and physical state during the flight. Previous studies [4, 5, 6, 7, 8, 9] also confirmed that pilots' psychophysiological parameters provide information about mental and physical condition.

Among the simplest and most commonly used methods of examining the individual's biological signals belongs electrocardiography. This method provides information on cardiac activity (especially regarding heart rate) from which it is possible to read and measure the basic psychophysiological parameters of a measured subject [10]. The heart rate desribes activity of the human heart and its psychophysiological character influenced by the sympatovagal balance of autonomic nervous system (ANS). More precisely the ANS sympathetic and parasympathetic components are activated. Sympathetic component stimulates cardiac action through nervi cardiaci, i.e. RR interval is shortened, and parasympathetic component cardiac action decreases through rami cardiaci from nervous vagus, i.e. RR interval is prolonged [11, 12].

Many foregoing studies [7, 8, 9, 13] processed measured data by standard methods. This paper is oriented towards description and comparison of the most common methods for physiological parameters assessment (from time domain, frequency domain and non-parametric methods) and also describes a wavelet transform, method that analyses heart rate signal both in time and frequency domain.

Wavelet Transform (WT) was developed at the end of the 20th century by evolving the Short Time Fourier Transformation (STFT). Wavelet transform is based on high frequency waves with limited time of its duration. It serves mainly to process non-stationary images and signals that can be analyzed in both frequency and time domains. Therefore, WT appears to be a suitable tool for spectral analysis of data with information on occurrence of individual phenomena. The greatest benefit of WT is its ability to analyze the signal locally with a variable scale, i.e. to adapt the characteristics of analysis to the character of the signal to obtain optimal settings. Wave transformation is also referred to as a multiscale analysis or a multiresolution analysis due to how it functions. It is mainly used for processing and evaluation of signals or data. Due to WT's properties it can be used for a decomposition of the signal on the base part or its compression to detect the position and the length of a given phenomenon or for a recognition of a discontinuity of signal. Its practical applications range in a wide spectra of areas. For this contribution it is beneficial to use it in the analysis of biosignals, especially the ECG, primarily with regard to the large number of minor fluctuations for which the time and frequency information must be known. In terms of the ECG signal WT can replace the standard spectral analysis by which power is obtained in the individual spectral areas. Added value of WT is time information about specific frequency changes [14].

Pilot's psychophysiological state data were obtained during measurements on predetermined flights on flight simulator and real airplane with analogue and then with digital visualization of flight data (Glass Cockpit).

\section{Materials and Methods}

\subsection{Participants and measurement procedure}

The group of participants was made up of 10 healthy pilots in training all with the same level of experience. The group consisted of 8 men and 2 women with an average age of $22 \pm 5$ years. The main prerequisite for being included into the study was successful completion of psychological and knowledge tests. Used psychological test created by the Institute of Aviation Health examined the emotional resilience of the candidates and their attention. The knowledge test was focused on knowledge of flight basics and aeronautical regulations. Furthermore, participants had to meet the criteria for medical fitness according to Annex IV and had to be without flight experience, i.e. participants could not hold a pilot license of any type (ULL, PPL or higher).

The measurements were made using a TRD40 type flight simulator and real airplane Diamond DA40. In both the simulator and the aircraft the analogue visualization of the flight and navigation data was as usual, the so-called "Basic-T" layout. In the case of digital visualization (Glass Cockpit) the cockpit dashboard was equipped with an integrated aircraft instrument system Garmin G1000. Real flights took place at Kosice International Airport (ICAO code - LZKZ). For reasons of consistency of measurement for future comparisons flights were conducted under the same meteorological conditions without or with low cloudiness and under standard flight conditions with a visible surface of the ground (VFR flight).

Each of the pilots also had to complete the prescribed route with predefined flight manoeuvres (Fig. 1) to ensure uniform conditions of measurement. The flight consisted of take-off, a three-time series of four manoeuvres - a straight level flight (SLF), horizontal $360^{\circ}$ turn (H360), climb $180^{\circ}$ turn (C180), descend $180^{\circ}$ turn (D180) and landing. The bank angle for the $\mathrm{H} 360$ was $30^{\circ}$, for the $\mathrm{C} 180$ and D180 was $15^{\circ}$ and the vertical speed of climb / descend equal was $500 \mathrm{ft} / \mathrm{min}$. All tasks were conducted by pilot under the supervision of an instructor who instructed the pilot to follow the plan. The average flight time from take-off to landing was $38.2 \mathrm{~min}$ ( $\mathrm{SD} \pm 4.4 \mathrm{~min})$.

The whole training consisted of 19 flight sessions with the series of manoeuvres described above repeated during each flight. Participants first took a flight simulation training session (11 lessons), then performed one real flight and then attended three additional training sessions on the simulator. All of training in a cockpit equipped with analogue display was ended with two real flying lessons. The last two lessons took place in digital flight data display (Glass Cockpit) cockpit. 


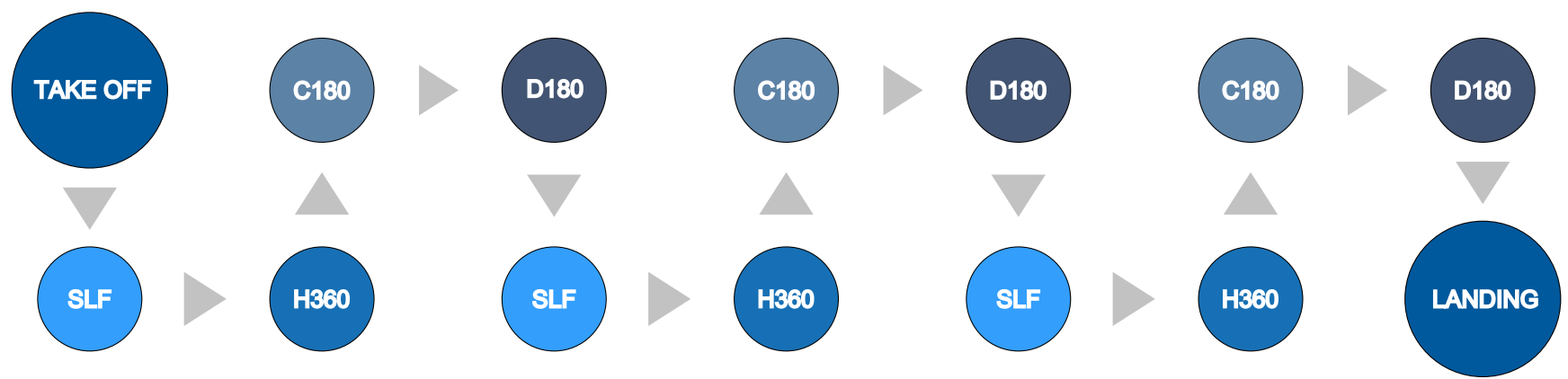

Figure 1. Predefined flight manoeuvres.

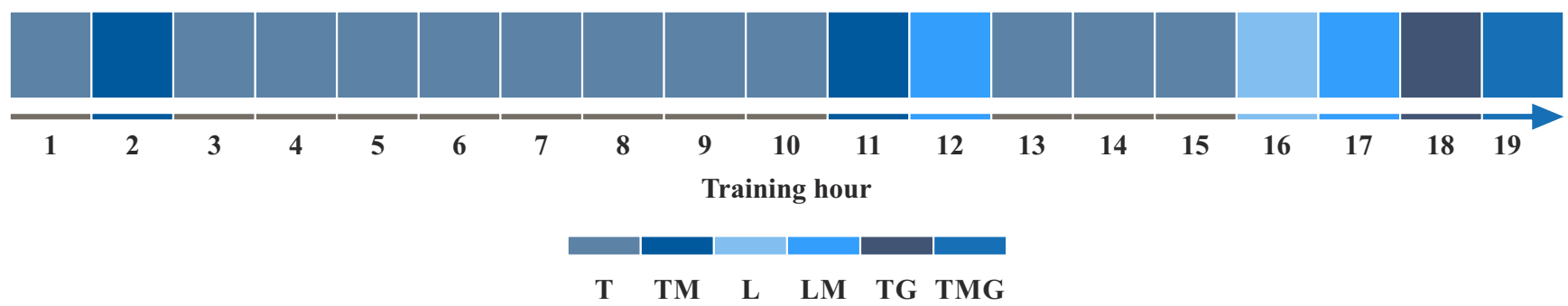

Figure 2. Distribution of measuring during the 19 flight lessons ( $\mathrm{T}$ - simulator, $\mathrm{TM}$ - measuring on simulator, $\mathrm{L}-$ real flight, LM - measuring in real flight, TG - simulator with Glass Cockpit, TMG - measuring in real flight with Glass Cockpit).

The pilot's heart rate measurement was recorded during the selected flights - on flights with an analogue display during the second (T2M) and eleventh (T11M) session on simulated flight and during the twelfth (L12M) and seventeenth (L17M) lessons on real flight in the aircraft. Glass cockpit measurements were recorded in both cases, on the simulator (T18M) and on the aircraft (L19M) (Fig. 2).

\subsection{Measuring equipment}

Due to advanced technological progressions a new form of remote human and animal health monitoring can be used the biotelemetric system. Mobile telemetry system of biological parameters serves for reading and wirelessly transmitting measured data of selected biological parameters to a computer or other electrical equipment. Most of these systems use internet, wireless communication protocols (e.g. Bluetooth) or wireless mobile networks to transfer measured data. A typical biotelemetry system includes sensors tracking specific signals, a battery, an antenna, a receiver and an imaging unit. It is about facilitating not only medical care but also other areas of life, for example in the sports, science and technology sectors and in all other areas where biosignals are being explored [15].

For the purpose of this paper pilot's psychophysiological parameters during each flight were measured by FlexiGuard (FG) system. FG is a portable telemetry system that monitors in real time physiological state of the pilot and the parameters of the environment in which the subject is currently located. The system consists of three basic units. The first component is a sensing unit (the sensor itself) that records desired values and then sends it via the wireless system to the second unit
- the central unit. It functions to collect data from sensors, store them and partially evaluate them. Finally, the data are sent via the ANT + wireless connection to the third part connected to the computer, to the visualization unit [16]. For the monitoring of heart rate telemetry system was equipped with a commercial chest belt by Garmin ${ }^{\mathrm{R}}$. The ECG sensing electrodes in the right and left zone of chest belt can recognize QRS then system can determine the heart rate and the number of RR intervals. The signal in the form of RR intervals over the measured time period was further evaluated by the selected methods.

\subsection{Physiological parameters evaluation methods}

Because of the non-linear disposition of autonomic neuronal regulation, only RR interval values were used to process heart rate data. The RR intervals was processed by the HRVAS, a heart rate variability (HRV) analysis tool developed using MATLAB. HRVAS can decompose and filter IBI (inter beat interval) and can perform time domain, frequency domain, time-frequency, Poincare and nonlinear HRV analysis. HRVAS also can convert measured data displayed only in a text format into numbers in a neat table where the individual measured values can be seen. The table consists of 4 rows and 105 columns. The table contains all measured physiological data about the individual during one flight, so it consists of information about the parameters from the frequency domain (see Tab. 1), the time domain (see Tab. 2) and from the nonlinear analysis (see Tab. 3). 
Table 1. Parameters in frequency domain.

\begin{tabular}{|c|c|c|}
\hline Parameter & Unit & Description \\
\hline ULF power & {$\left[\mathrm{ms}^{2}\right]$} & Absolute power of the ultra-low frequency band $(\leq 0.003 \mathrm{~Hz})$. \\
\hline VLF power & {$\left[\mathrm{ms}^{2}\right]$} & Absolute power of the very-low frequency band $(0.0033-0.04 \mathrm{~Hz})$. \\
\hline LF peak & [Hz] & Peak frequency of the low frequency band. \\
\hline LF power & {$\left[\mathrm{ms}^{2}\right]$} & Absolute power of the low frequency band $(0.04-0.15 \mathrm{~Hz})$. \\
\hline LF power & [NU] & Relative power of the low frequency band in normal units. \\
\hline LF power & [\%] & Relative power in low frequency band in percent. \\
\hline HF peak & {$[\mathrm{Hz}]$} & Peak frequency of the high frequency band. \\
\hline HF power & {$\left[\mathrm{ms}^{2}\right]$} & Absolute power of the high frequency band $(0.15-0.4 \mathrm{~Hz})$. \\
\hline HF power & [NU] & Relative power of the high frequency band in normal units. \\
\hline HF power & [\%] & Relative power in the high frequency band in percent. \\
\hline $\mathrm{LF} / \mathrm{HF}$ & {$[-]$} & Ratio of LF to HF power. \\
\hline
\end{tabular}

Table 2. Parameters in time domain.

\begin{tabular}{|c|c|c|}
\hline Parameter & Unit & Description \\
\hline SDNN & [ms] & Standard deviation of the NN intervals. \\
\hline SDRR & [ms] & Standard deviation of the RR intervals. \\
\hline SDANN & {$[\mathrm{ms}]$} & $\begin{array}{l}\text { Standard deviation of mean NN intervals for each five minute segments of } \\
\text { a } 24 \text { hours HRV recording. }\end{array}$ \\
\hline SDNN index (SDNNI) & [ms] & $\begin{array}{l}\text { Mean of the standard deviations of all the NN intervals for each five } \\
\text { minutes segment of a } 24 \text { hours HRV recording. }\end{array}$ \\
\hline pNN50 & [\%] & Percentage of succesive RR intervals that differ by more than $50 \mathrm{~ms}$ \\
\hline RMSSD & [ms] & Root mean square of succesive RR interval differences. \\
\hline HR $\max -\mathrm{HR} \min$ & [bpm] & $\begin{array}{l}\text { Average difference between the highest and lowest heart rates during each } \\
\text { respiratory cycle. }\end{array}$ \\
\hline $\begin{array}{l}\text { HRV triangular index } \\
\text { TINN }\end{array}$ & $\begin{array}{l}{[-]} \\
{[\mathrm{ms}]}\end{array}$ & $\begin{array}{l}\text { Integral of the density of the RR interval histogram divided by its height. } \\
\text { Baseline width of the RR interval histogram. }\end{array}$ \\
\hline
\end{tabular}

Table 3. Parameters in nonlinear analysis.

\begin{tabular}{|c|c|c|}
\hline Parameter & Unit & Description \\
\hline $\mathrm{S}$ & [ms] & Area of the ellipse which represents total HRV. \\
\hline SD1 & [ms] & Poincaré plot standard deviation perpendicular the line of identity. \\
\hline SD2 & [ms] & Poincaré plot standard deviation along the line of identity. \\
\hline SD1/SD2 & [\%] & Ration of SD1 to SD2. \\
\hline ApEn & {$[-]$} & Approximate entropy, which measures the regularity and complexity of a time series. \\
\hline SampEn & {$[-]$} & Sample entropy, which measures the regularity and complexity of a time series. \\
\hline DFA $\alpha 1$ & {$[-]$} & Detrended fluctuation analysis, which describes short-term fluctuation. \\
\hline DFA $\alpha 2$ & {$[-]$} & Detrended fluctuation analysis, which describes long-term fluctuation \\
\hline D2 & {$[-]$} & $\begin{array}{l}\text { Correlation dimension, which estimates the minimum number of variables required to } \\
\text { construct a model of system dynamics. }\end{array}$ \\
\hline
\end{tabular}

\subsection{Statistical analysis}

To determine the validity of each parameter, each of the parameters was subjected to statistical analysis based on the comparison of intergroup differences, where these groups represent the individual training phases (flight hours). One flight is composed of 12 manoeuvres for all subjects separately, i.e. one group represents 120 measurements characterized by the selected parameter in one flight hour. The standardization of the data was performed before unification of the results of individual subjects. The standardization was implemented in a form in which the distribution of calculated parameters from a single flight (manoeuvre) by a particular subject was unified to an average value equal to 0 and a standard deviation equal to \pm 1 . This procedure was realized to avoid inter-individual variability (for one subject during training) and intra-individual variability (between subjects).

Due to the fact that these are dependent measurements it was necessary to use double-test for statistical analysis. In addition, statistical testing was conditional on the distribution of test groups and therefore normality testing was performed. This test was carried out using a Jarque Bera test [17] which tests null hypothesis that the data under consideration origi- 
nates from a normal distribution compared to an alternative hypothesis stating that these data from normal distribution do not occur at a significance level alpha $=0.05$. For $p<0.05$, it is not possible to reject the zero hypothesis. In addition to the using the two-sample test, it is necessary to use non-parametric testing. For the above mentioned reasons Wilcoxon's twosample test was used for further statistical analysis. This test assumes that both selections with continuous distribution functions have the same probability distribution. This test is equivalent to a parametric two-sample test, but compares variance and expected value [18]. However, in this paper there are more than two measured files, therefore Bonferroni correction is needed. Bonferroni correction is used when it is necessary to compare three or more measured groups. If a significant difference in the test is needed Bonferroni correction will point out to pairs between where there is a statistically significant difference.

\section{Results}

The pilot's training data were processed using proposed methods in the time, frequency, time-frequency domain and nonlinear analyses. The distribution of the selected parameters throughout the training is presented in the form of boxplots. The Wilcoxon test was used to determine the statistical differences between phases. Due to the fact that 15 pairs of data were compared in a presented way the test was extended by Bonferroni's correction. In this section only two results are shown. This is the LF/HF ratio in the frequency domain (Fig. 3) and then in the wavelet transform (Fig. 4). The LF/HF is mentioned for its best expression of the pilot's psychophysiological state during the flights. Table 4 and 4) present $\mathrm{p}$ values for $\mathrm{LF} / \mathrm{HF}$ parameters.

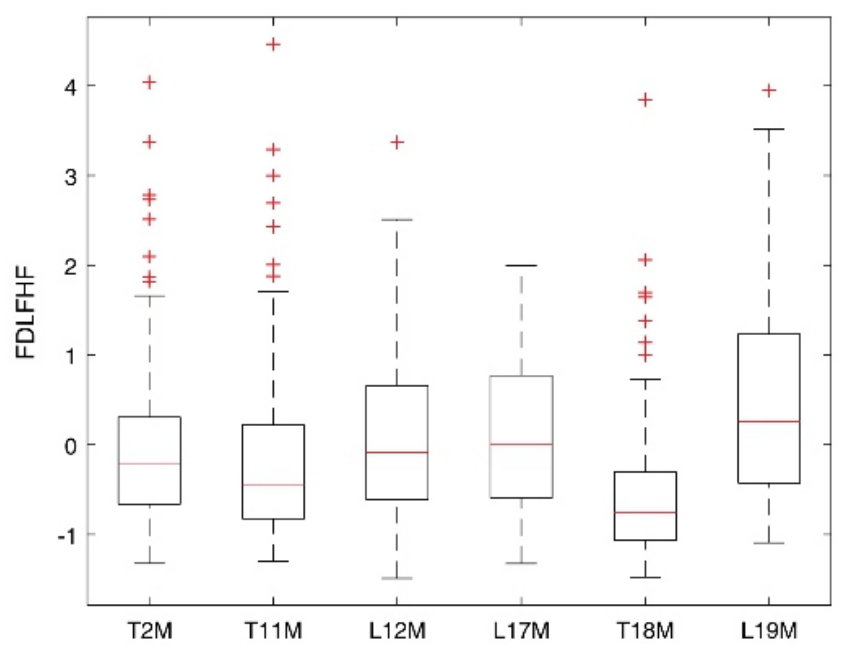

Figure 3. Boxplot of frequency domain parameter LF/HF ratio.
Table 4. P-value as a result of flight comparison for frequency domain parameter $\mathrm{LF} / \mathrm{HF}$ ratio.

\begin{tabular}{l|llllll} 
& T2M & T11M & L12M & L17M & T18M & L19M \\
\hline T2M & 1 & 0.04 & 0.27 & 0.06 & $<0.05$ & $<0.05$ \\
T11M & 0.04 & 1 & $<0.05$ & $<0.05$ & $<0.05$ & $<0.05$ \\
L12M & 0.27 & $<0.05$ & 1 & 0.52 & $<0.05$ & $<0.05$ \\
L17M & 0.06 & $<0.05$ & 0.52 & 1 & $<0.05$ & 0.02 \\
T18M & $<0.05$ & $<0.05$ & $<0.05$ & $<0.05$ & 1 & $<0.05$ \\
L19M & $<0.05$ & $<0.05$ & $<0.05$ & 0.02 & $<0.05$ & 1 \\
\hline
\end{tabular}

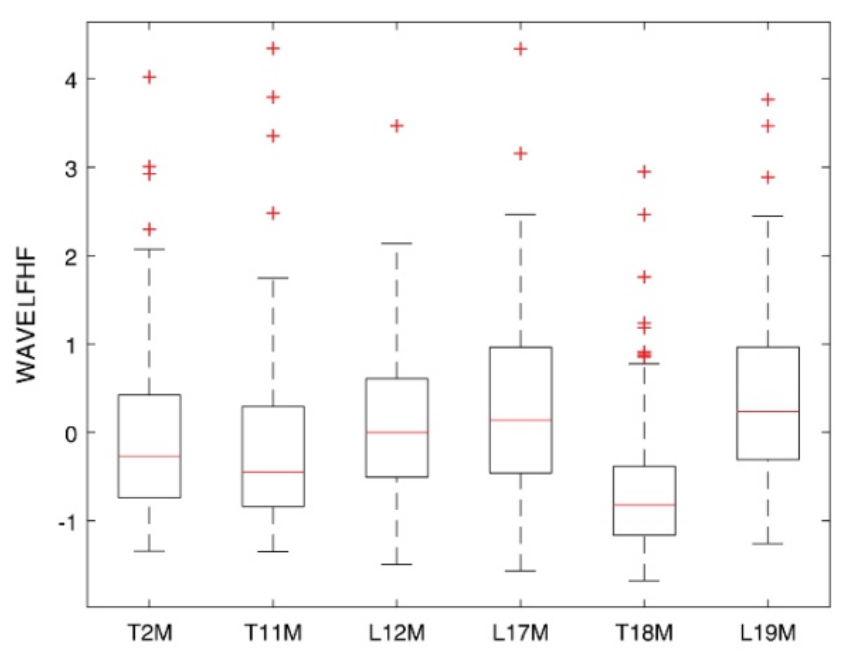

Figure 4. Boxplot of time-frequency domain parameter $\mathrm{LF} / \mathrm{HF}$ ratio

Table 5. $\mathrm{P}$ value as a result of flight comparison for time-frequency domain parameter LF/HF ratio.

\begin{tabular}{l|llllll} 
& T2M & T11M & L12M & L17M & T18M & L19M \\
\hline T2M & 1 & 0.18 & 0.05 & $<0.05$ & $<0.05$ & $<0.05$ \\
T11M & 0.18 & 1 & $<0.05$ & $<0.05$ & $<0.05$ & $<0.05$ \\
L12M & 0.05 & $<0.05$ & 1 & 0.12 & $<0.05$ & 0.02 \\
L17M & $<0.05$ & $<0.05$ & 0.12 & 1 & $<0.05$ & 0.48 \\
T18M & $<0.05$ & $<0.05$ & $<0.05$ & $<0.05$ & 1 & $<0.05$ \\
L19M & $<0.05$ & $<0.05$ & 0.02 & 0.48 & $<0.05$ & 1 \\
\hline
\end{tabular}

\section{Discussion}

For the purpose of the analysis standard methods have been chosen but these methods have many limitations, especially time domain analysis. For this reason, other approaches had also been chosen that should better present physiological data taking into account their nature. From the results presented in the previous chapter it is obvious that most of the selected parameters show the same trend - increasing workload during a new session and decreasing workload during repetitive session. Thus there is an increase and decrease of values according to certain types of flights - simulated or on an aircraft. More specifically, in LF/HF in the frequency domain and in the time frequency domain it is possible to see the difference between the measured values during various flight lessons. Although there is no statistically significant difference between 
$\mathrm{T} 2 \mathrm{M}$ and $\mathrm{T} 11 \mathrm{M}$ the overall sympathetic rate of subjects has decreased. Therefore, it can be assumed from a given measurement that after 10 lessons on the simulator the subjects calmed down and thus were under less stress.

For real flight measurements, the difference is not very obvious, on the contrary the L17M value appears to be greater than L12M. However, the deviation is subtle. The biggest difference between the measured values, however, is evident between the simulated Glass Cockpit flight (T18M) and the real Glass Cockpit flight (L19M). This difference indicates that the subjects were under greater workload when flying in airplane. After the decomposition of the signal over time it is possible to determine when the WT has exactly increased the load on the given subject, or it is possible to precisely define the time and hence the phase of the flight during which the load has increased to the psychophysiological condition of the measured individual. By using the WT for the decomposition of the signal over time it is possible to determine when exactly had workload of a measured subject increased.

Predicted trend of the presented parameters can be found throughout the training. Particularly in the wavelet transform analysis, this trend can be found with a large number of parameters, not only in $\mathrm{LF} / \mathrm{HF}$ ratio. Because of strictly set training, this trend should occur, so it can be assumed that these parameters can be considered appropriate for the analysis. Similarly, in assessing the similarity of individual flight phases wavelet transformation can generally find a number of significant transitions between individual flight phases. Based on the above mentioned facts, we can assume that wavelet transformation is usable as a credible tool for objectivizing aeronautical training. Other analyses, especially time domain analysis, were unable to analyse the data in the required way based on assumptions derived from the set training.

\section{Conclusion}

All analyses performed for the purpose of the presented research were carried out post-hoc, i.e. after the measurement of the complete flight data. In this respect, there is currently an important opportunity to upgrade research to the next level through online monitoring and assessment of the pilot's status and the course of its flight. In this case, the instructor should be provided with information on the change of psychophysiological status and the course of the flight immediately during the flight which could serve as an immediate feedback to the instructor. Consequently, the concept can serve as base for further research into the development of EBT and ATQP. Its benefits can therefore be utilized to streamline training which brings benefits in terms of reducing the cost of training itself and adjusting training for each individual subject on the basis of an objective (and subjective) assessment of pilots' skills.

Individualization of the training can lead to a deepening of pilots' knowledge and to reinforcement of his / her skills due to the elimination of redundant training in the area where the subject is well-trained and focusing on the problematic parts of training of the given subject, to shift individual training forward. However, the use of the concept itself requires further investigation in this regard. The limitation of research is smaller number of measured subjects who participated in the measurement. To further explore this issue, it is therefore advisable to increase the number of measured subjects and thereby create a larger database of results that can support the advantages of WT during flight.

\section{Acknowledgments}

This study was supported and cofinanced by APVV-17-0167 project "Application of the self-regulatory methods for the preparation of flight crew" (Agency for the Support of Research and Development of the Ministry of Education, Science, Research and Sports of the Slovak Republic) and SGS17 /150/OHK2/2T/16 project "Evaluation of psychophysiological state of pilots based on physiological parametrs" (Internal Grant Agency of Czech Technical University in Prague).

\section{References}

[1] European Aviation Safety Agency. European plan for aviation safety (epas): 2018 - 2022, 2017. URL https: / /www . easa.europa.eu/sites / default/files/dfu/EPAS_2018-2022\%20v2. $2.8 \div 20$ for $\% 20 \mathrm{MB}$.pdf. [Online].

[2] European Aviation Safety Agency. European plan for aviation safety: 2016 - 2020, 2016. URL https : / / www . easa.europa.eu/sites/default/files/ $\mathrm{dfu/EPAS} \div 202016-2020 \% 20 \mathrm{FINAL}$. PDF. [Online].

[3] ICAO. Accident Statistics, 2018. URL https: / / www.icao.int/safety/istars / Pages/Accident-Statistics.aspx. [Online].

[4] Glenn F Wilson and Frank Fisher. The use of multiple physiological measures to determine flight segment in $\mathrm{f} 4$ pilots. In Aerospace and Electronics Conference, 1990. NAECON 1990., Proceedings of the IEEE 1990 National, pages 859-861. IEEE, 1990.

[5] Malcolm A. Bonner and Glenn F. Wilson. Heart rate measures of flight test and evaluation. The International Journal of Aviation Psychology, 12(1):63-77, jan 2002. doi: 10.1207/s15327108ijap1201_6. URL https : / / doi.org/10.1207/s15327108ijap1201_6.

[6] P. Hoogeboom, M. Joosse, H. Hodgetts, S. Straussberger, and D. Schaefer. Does the "silent cockpit" reduce pilot workload? In The 23rd Digital Avionics Systems Conference (IEEE Cat. No.04CH37576). IEEE, 2004. doi: 10.1109/dasc.2004.1391346. URL https : //doi.org/10.1109/dasc.2004.1391346.

[7] V.Socha, L.Socha, J. Schlenker, K. Hána, L. Hanakova, B. Mihalčová A. Lalis, P. Smrcka, and D. Hůlek. 
Evaluation of pilots' psychophysiological condition using recurrence quantification analysis of heart rate variability. In Proc. Of Transport Means 2016, Juodkrante, Lithuania, 2016. Kaunas University. URL https: / / www . researchgate. net/publication/ 314152478_Evaluation_of_Pilots\%27_ Psychophysiological_Condition_Using_ Recurrence_Quantification_Analysis_ of_Heart_Rate_Variability.

[8] Marek Regula, Vladimir Socha, Patrik Kutilek, Lubos Socha, Karel Hana, Lenka Hanakova, and Stanislav Szabo. Study of heart rate as the main stress indicator in aircraft pilots. In Proceedings of the 16th International Conference on Mechatronics - Mechatronika 2014. IEEE, dec 2014. doi: 10.1109/mechatronika.2014. 7018334. URL https://doi.org/10.1109/ mechatronika.2014.7018334.

[9] Lenka Hanakova, Vladimir Socha, Lubos Socha, Stanislav Szabo, Jaroslaw Kozuba, Andrej Lalis, Peter Vittek, Jakub Kraus, Robert Rozenberg, Peter Kalavsky, Martin Novak, Jakub Schlenker, and Stanislav Kusmirek. Determining importance of physiological parameters and methods of their evaluation for classification of pilots psychophysiological condition. In 2017 International Conference on Military Technologies (ICMT). IEEE, may 2017. doi: 10.1109/miltechs.2017.7988810. URL https : / / doi.org/10.1109/miltechs.2017.7988810.

[10] Petr Heřman. Biosignály z pohledu biofyziky. Petr Heřman - Dúlos, Praha, 2006. ISBN 80-902899-7-5. In Czech.

[11] Radomir Cihak. Anatomie. Grada, Praha, 2001. ISBN 80-247-1132-X. In Czech.

[12] Fred Shaffer and J. P. Ginsberg. An overview of heart rate variability metrics and norms. Frontiers in Public Health, 5, sep 2017. doi: 10.3389/fpubh. 2017.00258. URL https://doi.org/10.3389/ fpubh.2017.00258.

[13] Andrea Haarmann, Wolfram Boucsein, and Florian Schaefer. Combining electrodermal responses and cardiovascular measures for probing adaptive automation during simulated flight. Applied Ergonomics, 40(6):1026-1040, nov 2009. doi: 10.1016/j.apergo. 2009.04.011. URL https://doi .org/10.1016/ j.apergo.2009.04.011.

[14] Paul Addison. The illustrated wavelet transform handbook : introductory theory and applications in science, engineering, medicine and finance. CRC Press, Taylor \& Francis Group, Boca Raton, FL, 2016. ISBN 9781482251326.

[15] M. Penhaker, M. Černý, L. Martinák, and J. Spišák. Biotelemetry system of humans. IFAC Proceedings
Volumes, 39(21):406-411, feb 2006. doi: 10.1016/ s1474-6670(17)30220-3. URL https:// doi.org/ $10.1016 / \mathrm{s} 1474-6670$ (17) 30220-3.

[16] Jakub Schlenker, Vladimir Socha, Pavel Smrcka, Karel Hana, Vladimir Begera, Patrik Kutilek, Zdenek Hon, Jan Kaspar, Lukas Kucera, Jan Muzik, Tomas Vesely, and Martin Viteznik. FlexiGuard: Modular biotelemetry system for military applications. In International Conference on Military Technologies (ICMT) 2015. IEEE, may 2015. doi: 10.1109/miltechs.2015.7153712. URL https : / / doi.org/10.1109/miltechs.2015.7153712.

[17] Keya Rani Das. A brief review of tests for normality. American Journal of Theoretical and Applied Statistics, 5(1):5, 2016. doi: 10.11648/j. ajtas.20160501.12. URL https://doi.org/10. $11648 / j . a j t a s .20160501 .12$.

[18] Elise Whitley and Jonathan Ball. Statistics review 6: Nonparametric methods. Critical Care, 6(6):509, 2002. doi: 10.1186/cc1820. URL https: / / doi . org/10. $1186 / \operatorname{cc} 1820$. 